\title{
Low-cost-high-accuracy yard equipment tracking for improved container monitoring and assignment
}

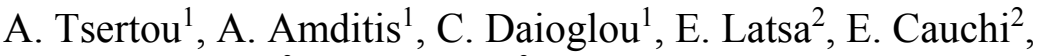 \\ I. Kanellopoulos ${ }^{3} \&$ M. Kotras ${ }^{3}$ \\ ${ }^{1}$ I-SENSE Group, Institute of Communication and \\ Computer Systems, Greece \\ ${ }^{2}$ SEAbility Ltd, Greece \\ ${ }^{3}$ Piraeus Container Terminal, Greece
}

\begin{abstract}
The INTE-TRANSIT management system (ITMS) is a low-cost, high-accuracy client-server system, suitable for improving the port efficiency through an enhanced monitoring of containers and of yard equipment (trucks, straddle carriers, reach stackers). The main purpose of the proposed system is to obtain information such as container identification, vehicle/yard equipment ID and positioning data from communication endpoints, namely Differential GPS receivers, RFID readers as well as GEO-location servers. The objectives are the accurate management of the container storage process inside the terminal at a minimal cost and an always up-to-date inventory of the stored containers. These functionalities are combined with a user-friendly visualization tool for yard equipment positioning and jobs, which offers various mapping capabilities, a container search functionality and real-time remote access. To meet such requirements, ITMS consists of well-defined layers of operation; each operates independently, while also being able to communicate with its neighbouring layers, even under heavy workloads. On the other hand, the client terminal is a device which features a software-RTKLIB based positioning device, a controller to an RFID reader, integrated to a low-cost processor and a user interface for driver. The operation and functionalities of the proposed system have been showcased in a pilot demonstration which has been performed in the Piraeus Container Terminal, the fastest growing port terminal in Europe in 2013.
\end{abstract}

Keywords: port terminal, DGPS, RFID, container monitoring, yard equipment. 


\section{Introduction}

Mediterranean (MED) port terminals are major logistic hubs for the circulation of goods, not only within the MED basin but also connecting European countries with key Asian ports. The efficiency of operations inside the port terminal as well as the connection of the terminal to associated warehouses and logistics areas are a constant challenge for the port operations personnel. The INTETRANSIT project [1] aims to assist terminal operators address this challenge through the use of modern Information Communication Technologies (ICT); the latter are deployed to improve the ability of the port terminal in having an accurate and almost real-time monitoring mechanism for containers as well as yard equipment (trucks, reach stackers, straddle carriers etc.). The basic ICT technologies which are considered in the framework of INTE-TRANSIT are the differential GPS (DGPS) technology which achieves a sub-meter accuracy in determining the position of the containers and yard vehicles, not achievable by legacy COTS (Custom Off The Shelf) GPS receivers. Moreover, Radio Frequency Identifier (RFID) technology is used for container identification so that manual intervention is minimised in the container transfer and storage processes.

These ICT technologies have in general been considered in prior work, both in research papers and also deployed in a number of port terminals in Europe and beyond. In the work of Yuan and Huang [2], a four-layer architecture is presented, consisting of a data collection, RFID (Radio Frequency Identifier) network filter, RFID network process and business application layers; these provide the proposed system the ability to trace the container movement in real time using a network of RFIDs. The system is tailored for tracking containers along the supply chain, starting from the manufacturer's side by registering the EPC (Electronic Product Code) and ending within the distributor or retailer's EPCIS (Information Services) gets updated that the product has arrived. Moreover, Ting et al. [3] describe a case study where RFID equipment for vehicle tracking inside the yard terminal is evaluated for different ranges, velocities and penetration levels (e.g. near containers as opposed to in free, open areas); several benefits of such a COTS (Custom-Off-The-Shelf) system were showcased, such as better operation management, tracking driver behaviour, as well as assisting yard equipment routing inside the terminal area. Moreover, in Li et al. [4], a vehicle terminal system is proposed, composing of an ultra-low power 16-bit microcontroller, an acquisition unit of RFID tags information, a GPS receiver, a GSM module and the HMI (Human Machine Interface); the system is relatively low-cost, yet exhibits the accuracy limits of COTS GPS receivers and also communicates with the back-end through GSM short messages.

Large as well as smaller ports world-wide have implemented spatial information systems and information services which combine interoperable technologies. Such ports indicatively include, but are not limited to: Antwerp (Belgium), Chiwan Container Terminal/Shekou Container Terminal/Yantian International Container Terminal (P.R. of China), Buenaventura Container 
Terminal (Colombia), Grand Port Maritime de Marseille (France), COSCO-HIT (Hong Kong), Busan (Republic of Korea), ECT Rotterdam (the Netherlands).

As specific examples, the Port of Rotterdam (PoR) is the busiest port in Europe with 11.6 million TEU handled in 2013 [5]. The PoR has implemented information services which combine interoperable technologies such as AIDC (Automatic Identification and Data Collection), barcodes, GPS/DGPS, passive and active RFID, so as to enable and manage the monitoring of ships, trucks and other means of container-carrying transport, including AGVs (Automatically Guided Vehicles). Related information is available real-time over the Internet. In addition, a wealth of spatial information data of the PoR is also available on the Internet, aiming at providing general geographic information for all spatial objects and for all other geographically important articles in the harbour [6].

On a smaller scale, Associated British Ports' operation at Immingham on the Humber, with a current annual throughput of 200,000 TEU, has taken advantage of DGPS to make its container terminal work harder; to move more cargo in less time; and to do it in a way that protects the safety of employees. The level of real-time and accurate operational data has enabled the port to improve vessel turnaround times as well as container lifts per hour, whilst enjoying augmented safety as a result of far fewer manual tallies of, e.g., the container stack. Cuttingedge DGPS automation gives the port more lifts per hour and faster ship turnaround without increasing the footprint of the terminal [7].

The remainder of the paper is organised as follows: Section 2 describes the overall INTE-TRANSIT Management System (ITMS) architecture and server implementation, emphasizing on the web-based user interface; Section 3 describes the hardware and software developed for the INTE-TRANSIT client terminal, an integrated device which performs the exchange of communication messages with the server side, hosts the controllers of the DGPS and RFID reader as well as offers a lightweight user interface for the yard equipment driver. Moreover, the basic principles of software-based correction of the GPS signal are explained. Subsequently, in Section 4 the pilot demonstrations of the ITMS are described, with results as far as the performance of the system is concerned. The paper concludes with Section 5, where a summary of the proposed system is outlined as well as potential improvements/extensions.

\section{INTE-TRANSIT Management System architecture and server implementation}

The INTE-TRANSIT management system (ITMS) is a low-cost, yet highaccuracy client-server system, suitable for improving the efficiency of port terminals, such as the Piraeus port terminal, through an enhanced monitoring of the containers as well as of the yard equipment (trucks, straddle carriers, reach stackers, etc). Its ultimate objectives are an efficient and accurate management of the storage procedure accuracy of containers inside a port terminal area at a minimal cost and an always up-to-date inventory of the stored containers. These functionalities are combined with a user-friendly visualization tool for yard equipment positioning and jobs, which offers various and configurable mapping 
capabilities, a container search functionality and remote access for the system operator in almost real-time.

In order to meet the aforementioned requirements, the ITMS consists of several well defined layers of operation; each of these layers of operation should operate independently, while also having the ability to robustly communicate with its neighbouring layers, designed to ensure efficiency and reliability under heavy workloads. The architecture layers are described in what follows:

- A Middleware gateway is designed to maintain all information exchange activities between the web based user interface and the core software of the ITMS through the utilization of secure web service calls.

- A core software agent implements the "business" logic of the ITMS, maintains a connection to a Data Base Management Server (DBMS) in order to store and manage container and yard equipment positioning and identification information. It also implements all the required communication interfaces for the exchange and translation of data between all data sources (RFID, DGPS, DBMS, Graphical User Interface GUI).

- The hardware management endpoints include all the client software required to maintain the interconnection between the hardware elements that retrieve information (DGPS receivers, RFID readers) and the core software of the ITMS.

- The ITMS server GUI has been developed in such a way so as to provide the end-user with useful information and the same time to be user-friendly in its usage. For that reason, the GUI has been organized into five sections:

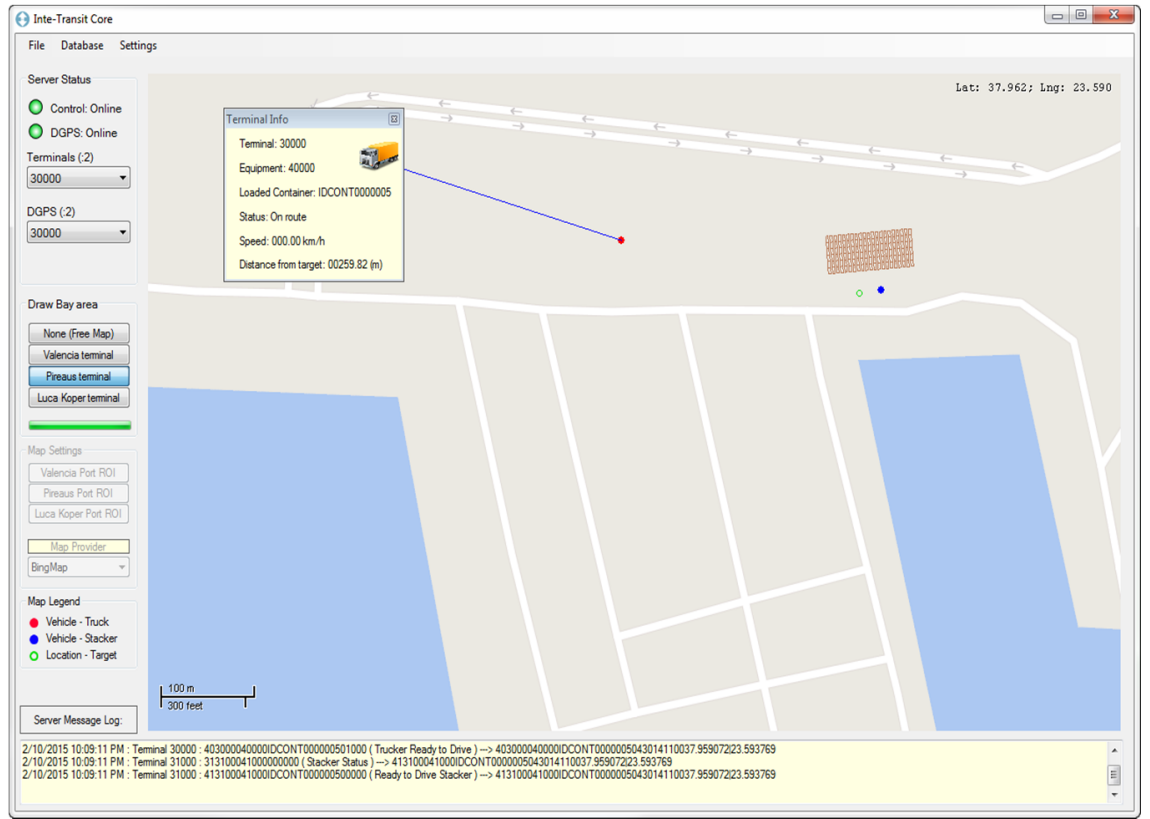

Figure 1: The INTE-TRANSIT management server GUI in Piraeus pilot. 
- The "Connectivity Status" area: In this area the user is being provided with information about the current network connection status of the server. Both the Control and DGPS modules are referenced in this area. Also, in this section the server provides the user with information about the connected client terminals as well as the connected DGPS modules. The user has the option to select between the connected client terminals and view specific information about a particular vehicle.

- The "Drawing Bay" and the "Map Settings" area: In this area the Server provides the user with the options of drawing the Bay Areas of the selected Container Terminal as well as moving the GIS Map's view to that particular Terminal. The drawing procedure of Bay Areas is based on the actual coordinates of every Bay, giving maximum viewing and calculating accuracy.

- The "GIS Mapping" area: In this area the server displays the GIS Map where the current activity of the ITMS is displayed. Every connected vehicle is spotted on the GIS Map in real-time and the user is provided with information about a vehicle's current job status, location, assigned container etc.

- The "Message Log" area: In this area, the server keeps a log file with information about the exchanged communication protocol frames between the server and all connected clients. The operator of the ITMS server may hide this area or make it visible depending on whether they want to operate it in debugging mode or in a normal operations mode.

- The "Container Search" area: This feature gives the ability to an administrator user to collect information about a specific container through the ITMS Server's GUI and can be used for container planning, tracing and monitoring purposes. The Container Search engine is implemented through an interactive form where the user has the following options:

Apply Search Filters: The user is given the option to apply search filters based on their needs. Depending on the applied filters the search engine will make queries from different points of the database (i.e. stored or pending containers). Furthermore, the search engine can make queries for currently assigned/on-going containers.

Enter a keyword: The search engine asks the user for a specific keyword to look for (i.e. a container ID).

Search by Container or Location ID: The user is also given the option to search either by Container ID or by Location ID. In this way the search engine is more flexible and the user can retrieve information like which container is stored (or is to be stored) at a specific location of the Bay Area. 


\section{INTE-TRANSIT client terminal}

The ITMS client terminal is responsible for connecting and collecting data from the various hardware components and peripherals of an ITMS-enabled vehicle and also for guiding the driver through the storage procedure of a container.

\subsection{Hardware equipment}

The equipment which has been used for the client terminal is listed in Table 1.

Table 1: Hardware equipment for INTE-TRANSIT client terminal.

\begin{tabular}{|c|c|}
\hline GPS receiver & $\begin{array}{c}\text { Denga10 } \\
\end{array}$ \\
\hline RFID reader & 2 USB-serial chips (FT230X) to access serial ports of the \\
& GNSS receiver (NMEA and BINR) \\
& SCIEL Reader R \\
& 433MHz Active RFID reader \\
Rs232/Rs485/USB interface \\
Wiegand 26bits, data and clock \\
High range: up to 200m, adjustable by software \\
IP65 waterproof \\
\hline RFID tag & Coin 1D, Tag SCT 01 \\
\hline Wi-Fi card, antenna & Wistron CM9 Atheros 802.11 a/b/g \\
\hline External antenna & Alfa Network 2.4 MHz Omni Indoor \\
(for base station) & Interline Horizon Omni Antenna 9dpi \\
\hline User terminal & Rugged Tablet PC M970D (OS: Windows) \\
\hline
\end{tabular}

A simple schematic that depicts the logical diagram of the client terminal is shown in Figure 2.

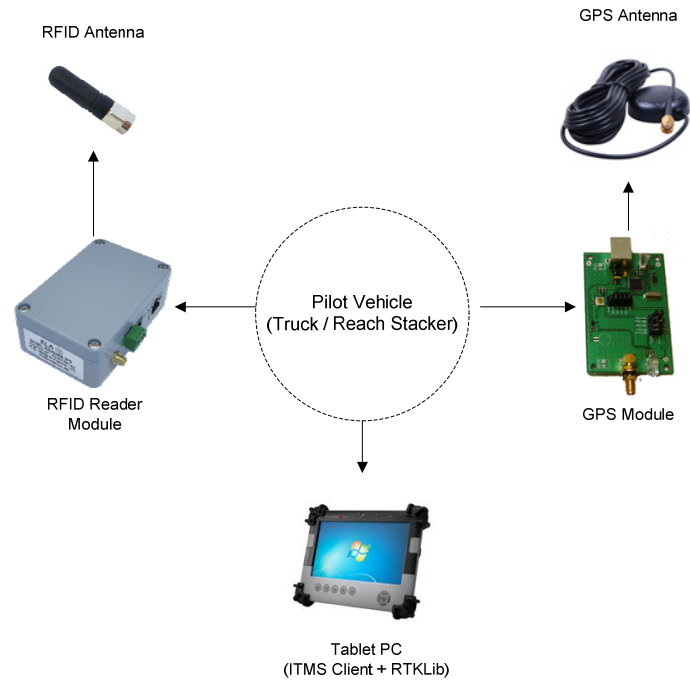

Figure 2: Logical diagram of the ITMS client terminal. 


\subsection{The RTKLIB library}

RTKLIB is an open source program package for standard and precise positioning with GNSS [8]. It has a portable program library and several APs (Application Programs) utilizing the library. It supports standard and precise positioning algorithms with GPS, GLONASS, GALLILEO, BEIDOU as well as various positioning modes with GNSS for both real-time and post-processing. The implementation chosen may use serial, TCP/IP, local log file or even FTP/HTTP for communication with the host-PC. The specifications of the Denga10 DGPS receiver which is RTKLIB compatible include a less than $30 \mathrm{sec}$ time to first fix and an accuracy in differential mode less than 1 meter. In our configuration, two receivers are used. One is located at an accurately surveyed point ("Base unit" or "Base station") and the other (usually referred to as the 'Rover unit') is located at the moving yard equipment (e.g. at the truck or at the reach stackers). Both units receive satellite data from a compilation of 'active' satellites as is the normal case with GPS. However, on top of that, through a network existing between the Base unit and the Rover Unit (in our case here this is a WiFi/IEEE 802.11 connection, but it could also be a GPRS connection or other), the base unit may calculate the correction to its position by comparing its known location (which is precisely defined) with the location determined by the GPS satellites. Then, the correction is transmitted to the rover unit and applied (through the hosted RTKLIB software) to the latter's calculated position.

\subsection{Software modules and functionalities}

The ITMS Client software is developed in an open-modular and flexible platform allowing the processing and handling of multiform data and consists of two key components:

- The client GUI which offers an interface with the end-user;

- The modules that implement all of the ITMS functionalities.

The ITMS Client GUI (see Figure 3) has been implemented as an interactive windows form where the user is informed about various parameters, conditions and instructions during an assigned job, such as:

- Real-time map monitoring and positioning of the vehicle.

- Real-time monitoring of current job, speed, container and bay target location.

- Interactive GIS-mapping with pan and zoom capabilities.

- Selective drawing of the Port bay area's through actual coordinates.

- Informative pop-up messaging when the vehicle accomplishes a job. 


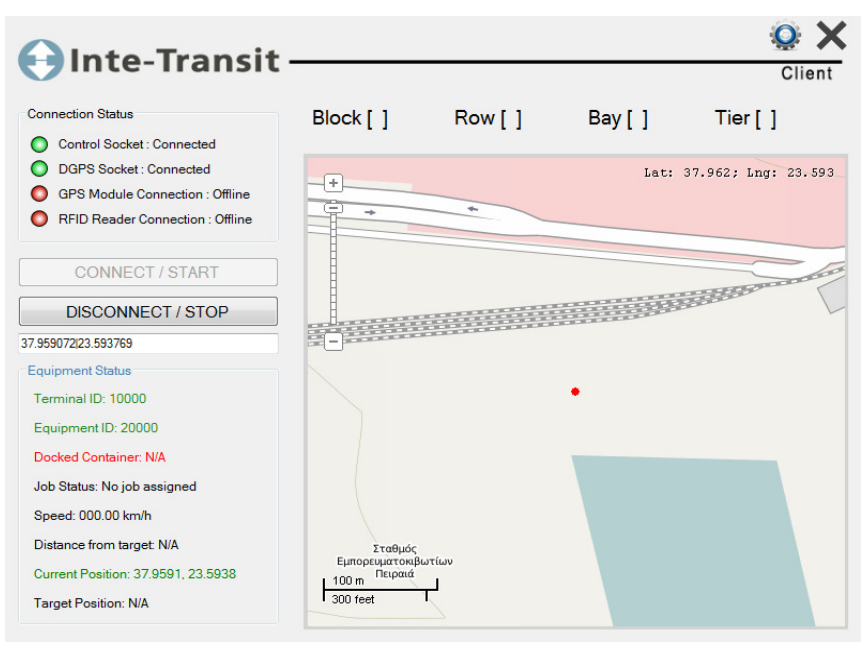

Figure 3: User interface of the client terminal.

All above information is in tight connection with the data acquired from the hardware equipment as well as the ITMS server control messages and the process algorithms of these data. Therefore, the client's core software consists of the following modules:

The RFID module: This module is responsible for communicating with the RFID Reader device. It is implemented through the serial protocol (RS232) and provides the client with the container ID loaded onto the vehicle.

The DGPS module: The DGPS module utilizes a local network TCP connection with the RTKLib and collects data about the vehicle/container's position. The data are in the form of the GPS/WGS84 datum and according to a proprietary, custom-made for INTE-TRANSIT communication protocol are formatted as "Latitude|Longtitude".

The TCP/IP (network) module: This module is responsible for connecting and communicating with the ITMS Server. It is implemented through remote network TCP connections for sending/receiving control messages and GPS position to the Server.

\section{Pilot description and demonstration}

\subsection{Piraeus Container Terminal and pilot description}

Piraeus Container Terminal SA is a wholly-owned subsidiary of COSCO Pacific Limited. It is principally engaged in the development, operation and commercial utilization of the Piraeus Container Terminal. It currently operates Pier II and III with a total capacity of $4.8 \mathrm{M}$ TEUs per year. It has recently initiated construction works for the west-bound expansion of Pier III that will increase the annual capacity to $6.7 \mathrm{M}$ TEUs per year seeking to become the leading port in the MED area. 
In PCT, ICT is considered a vital factor for the port development and has thus participated in the INTE-TRANSIT project as the leader of Pilot Group A. Pilot Group A of INTE-TRANSIT focuses on the improvement of monitoring and tracing containers and yard equipment. In detail, related functionalities include:

- Tracking of yard equipment.

- Localization of containers.

- Illustration of containers on the yard map

- Capturing of yard equipment data for further analysis

These functionalities have been achieved by the implementation of the ITMS which aims to monitor the position of yard equipment in the terminal yard and store the captured data for further analysis. The DGPS receiver is installed on monitored yard equipment that enables capturing yard equipment data related to the routes followed, idle times, total distance travelled loaded/unloaded etc. Moreover, an RFID reader is installed on the trucks participating to the pilot that provides the ability to capture container specific data by reading RFID tags mounted on the containers. By combining the location information coming from the DGPS receiver and the identification information coming from the RFID reader, the ITMS will be able to monitor the actual position of the containers in the yard.

The ITMS is also loaded with the planned container positions coming from the port's Terminal Operating System (TOS). This fact enables the matching of the actual position versus the planned one. In case of discrepancies, the client terminal installed on the yard equipment vehicle mounted terminals, will pop-up a warning message to the equipment driver informing him of the discrepancy. From that point on, the driver can decide whether to update the planned with the real position and inform their control tower accordingly or move the container to the planned position.

The implementation of the pilot in Piraeus has been performed using the current cloud infrastructure of PCT SA. A separate Class C VLAN (Virtual LAN) has been created to accommodate the ITMS along with the data monitoring stations. A separate SSID (Service Set Identification) has also been created for the accommodation of WiFi clients transmitting truck monitoring data back to the INTE-Transit Management server. A second Class C VLAN has been associated with the new SSID and traffic is routed through the wireless controller and over the central Layer3 switches of PCT SA. The data captured, are stored on the Central SAN (Storage Area Network). Long term storage is vital for the project since consumption trends and driving styles over time can reveal opportunities for fuel consumption and exhaust reductions. Initial data have shown a potential of $15 \%$ savings on yard trucks.

\subsection{Piraeus pilot scenarios and demonstration}

The scenarios that have been implemented and tested in the pilot activities are the following:

- New container insertion and registration to the server's database;

- Container assignment to a truck vehicle and transport to Bay area;

- Container assignment to a reach stacker vehicle and storage. 
The above scenarios involve extended testing of the ITMS server and client functionality in conjunction with the hardware equipment installed to the pilot vehicles and their corresponding assignments:

- The ITMS server software and its modules is installed and running under a windows-based computer with access to the existing network infrastructure of PCT.

- The ITMS client software is installed and running under a windowsbased tablet or rugged portable $\mathrm{PC}$ which is placed inside the corresponding pilot's vehicle.

- The DGPS base station serves as a reference point to a known geographical point. It is implemented through a GPS receiver connected to a windows-based computer at the pilot site running the RTKLib software. The data collected from the RTKLib are provided to every ITMS Client through the existing network infrastructure.

- The installation of client terminal hardware described in Table 1 on the vehicles of the yard (1 truck and 1 reach stacker, RS).

The scenario execution at the Piraeus pilot is according to the following map diagram: According to Figure 4, a truck vehicle (red line) is loaded with a container and requires assignment from the ITMS server. The server assigns the job to the truck and instructs to transport the container to the intermediate location pointed by the green circle.

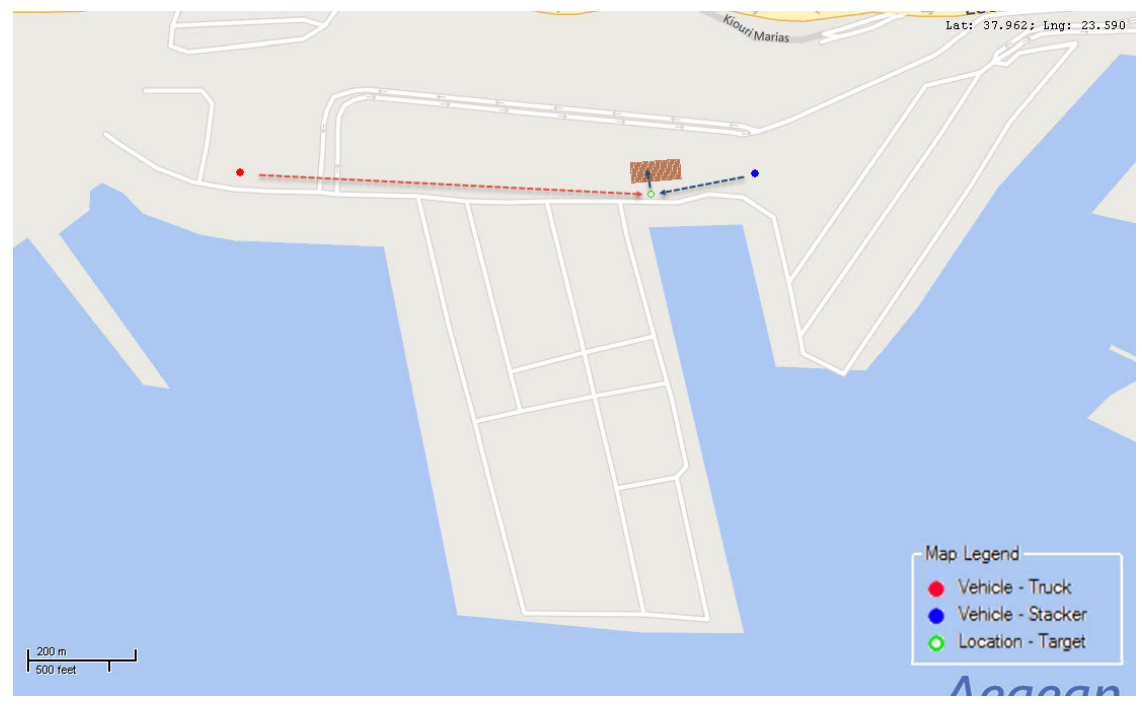

Figure 4: Schematic diagram of Piraeus pilot scenario.

At the same time a RS (blue line) requires assignment by the server, which on turn instructs it to move to the intermediate location (green circle). When the 
truck reaches the designated location the server will respond by informing the corresponding client that it reached its destination and wait for the RS to arrive. The RS client terminal will also be informed by the server when it arrives at the meeting location. At this point the RS will unload the container from the truck and the stacking procedure will be initiated by the server. When the container is positioned above the designated stacking location (brown-marked area) the server will inform the client and the vehicle's user will store the container at this bay. Both vehicles are released by the server when their corresponding job is successfully completed.

The above scenario has been executed with a successful bidirectional communication between the ITMS server and the vehicles client terminals.

Each client's job execution is logged by the server for further analysis according to the message format which has been defined as per the pilot requirements:

01/30/2015 2:03:37 PM: Terminal 10000: 100100000000000 ( Trucker Error )

01/30/2015 2:03:38 PM: Terminal 10000: 301000010000IDCONT2XXXXXX01000 ( Trucker Status )

01/30/2015 2:03:44 PM: Terminal 10000: 401000010000IDCONT2XXXXXX01000 ( Trucker Ready to Drive )

01/30/2015 2:03:58 PM: Terminal 10000: 501000010000IDCONT2XXXXXX23H00220100 ( Container Undocked )

01/30/2015 2:04:37 PM: Terminal 60000: 316000060000000000 ( Stacker Status )

01/30/2015 2:05:00 PM: Terminal 60000: 416000060000IDCONT2XXXXXX00000 ( Ready to Drive Stacker ) 01/30/2015 2:05:36 PM: Terminal 60000: 516000060000IDCONT2XXXXXX23H00221100 ( Stacker Reached Location )

01/30/2015 2:05:37 PM: Terminal 60000: 1116000060000IDCONT2XXXXXX23H00221000 ( Stack Location Ack )

01/30/2015 2:07:03 PM: Terminal 60000: 716000060000IDCONT2XXXXXX23H00220120 ( Container Stored )

\section{Conclusions}

The primary objectives of container tracking are physical localisation and terminal process optimisation. Nowadays, this inspection is mainly performed by human operators. In the INTE-TRANSIT project, the hardware solution designed for the client terminal in the Piraeus Port pilot was implemented, based on a lowcost implementation of (D)GPS and RFID technologies. On the other hand, the software architecture including all architectural elements of the ITMS designed for the purposes of INTE-TRANSIT was presented in detail, illustrating the open and modular system design principles followed. The main purpose of the ITMS is to obtain-retrieve data (container identification, truck id information, positioning data, control actions status) from all communication endpoints such as DGPS receivers, RFID readers; managing the storage procedure of a container inside the storage areas of the port authorities and also keeping a detailed inventory of the stored containers while also providing the ability to a remote user having all this information properly displayed and visualized inside a GUI.

\section{Acknowledgement}

This work is supported from the European Union, ERDF MED Programme under the INTE-TRANSIT project (Ref: 5187 | 2C-MED12-05 | Version: 5). 


\section{References}

[1] The INTE-TRANSIT project, http://www.inte-transit.eu

[2] Yuan, Zhengwu and Huang, Dongli. 2008. "A Novel RFID-based Shipping Containers Location and Identification solution in Multimodal transport". Paper presented at the CCECE/CCGEI, Niagara Falls, Canada, May 5-7.

[3] Ting, S.L., L.X. Wang and W.H. Ip, 2012. "A study on RFID adoption for vehicle tracking in container terminal". Journal of Industrial Engineering and Management, 5(1):22-52, doi: 10.3926/jiem.412.

[4] Li Huiping, Yi Zhou, Lu Tian, Chunlin Wan, 2010. "Design of a Hybrid RFID/GPS-Based Terminal System in Vehicular Communications". Paper presented at the $6^{\text {th }}$ International Conference on Wireless Communications, Networking and Mobile Computing (WiCOM), Chengdu, China, 23-25 September.

[5] http://www.statista.com/statistics/264171/turnover-volume-of-the-largestcontainer-ports-worldwide/ (Accessed 04 February 2015).

[6] Bonaca, J., Černjul, R., Vaclavek, S. (2013): Sustavi za upravljanje kontejnerskim terminalima podržani GNSS-om i GIS-om (Systems Management at container terminals supported by GNSS and GIS, Eccentric, no. 16 , pp. 72-75).

[7] Differential GPS improves Humber return on investment. http:/www.portstrategy.com/news101/port-operations/cargo-handling/ differential_gps_improves_return_on_investment_for_humber_port, accessed 05 February $201 \overline{5}$.

[8] RTKLIB: An open Source Program Package for GNSS positioning, http://www.rtklib.com/, accessed 06 February 2015. 